\title{
From trench fever to endocarditis
}

\author{
P Mann, F Nye, G Williams, A Walker, A Amadi
}

Postgrad Med J 2003;79:655-656

The case of a 44 year old woman with infective endocarditis due to Bartonella quintana, an organism long recognised to cause a condition known as trench fever, is reported. This case illustrates the lengthy differential diagnosis of "culture negative" endocarditis. In addition the presence of serological cross reactivity of Bartonella spp and Chlamydia spp demonstrates the potential for misdiagnosis in these circumstances.

B acteria of the genus bartonella are being increasingly seen as human pathogens. Currently 10 members of the genus have been identified, three of which have been implicated as causes of infective endocarditis in man. We report the case of a woman with bartonella infection.

\section{CASE REPORT}

A 44 year old woman presented to our centre with an 18 month history of generalised fatigue and malaise associated with a three day history of pleuritic chest pain, migratory arthritis, night sweats, and fever. The patient had lost approximately two stone $(12.7 \mathrm{~kg})$ in weight over the last year but denied any associated gastrointestinal symptoms. She rarely drank alcohol and there was no history of foreign travel or contact with domestic animals. The patient was employed as a supermarket cashier and there was a history of exposure to bird droppings at her work staff entrance.

On examination she was pyrexial $\left(38.6^{\circ} \mathrm{C}\right)$, and a degree of left axillary lympadenopathy was noted. Pulse rate was 110 beats/min and of high volume. Blood pressure was 120/ $90 \mathrm{~mm} \mathrm{Hg}$. Both heart sounds were audible with an additional loud ejection systolic murmur and a soft early diastolic murmur at the left sternal edge. A $4 \mathrm{~cm}$ firm, tender hepatomegaly and a 4-6 cm splenomegaly were palpable.

Baseline blood tests showed pancytopenia, haemoglobin was $73 \mathrm{~g} / \mathrm{l}$, platelets $104 \times 10^{9} / \mathrm{l}$, and white cell count $2.4 \times$ $10^{9} / \mathrm{l}$. Inflammatory markers were raised (C-reactive protein $120 \mathrm{mg} / \mathrm{l}$ and erythrocyte sedimentation rate (ESR) $64 \mathrm{~mm}$ in the first hour); renal profile and liver function tests were normal. Electrocardiography and chest radiography were normal.

At this stage the differential diagnosis was lengthy and included infective endocarditis, pulmonary embolism, lymphoma, and a connective tissue disorder. After blood cultures the patient was started on parenteral benzylpenicillin, augmentin, and gentamicin. A subsequent lung ventilationperfusion scan was normal. Urgent transthoracic echocardiography demonstrated a large aortic valve vegetation with moderate-severe aortic regurgitation (see fig 1).

Initial blood cultures were negative, but based on the echocardiographic findings a working diagnosis of infective endocarditis was made.

The patient's condition failed to improve over the next five days and the pyrexia persisted despite antibiotic treatment. Her clinical course was further complicated by a worsening of her neutropenia, which was felt to be related to a penicillin induced antibody complex phenomenon. Her antibiotics were changed to parenteral imipenim, ciprofloxacin, and oral fluconazole. Bone marrow and axillary lymph node biopsy specimens showed only reactive changes and cultures were negative. Abdominal and thoracic computed tomography demonstrated hepatosplenomegaly; no focal lesion or lymphadenopathy was seen.

Serial blood cultures remained negative and inflammatory blood markers raised. An autoantibody screen was positive for antinuclear antibody (1:40) and double stranded DNA at $159 \mathrm{IU} / \mathrm{l}$ (normal range 0-102 IU/1). Serology (complement fixation testing) was positive for chlamydia, at this stage oral doxycycline was added to the treatment. Immunofluorescence demonstrated high IgG antibody titres against Chlamydia psittaci and C pneumoniae. Blood sample polymerase chain reaction (PCR) testing was negative for Chlamydia spp and Bartonella spp.

Approximately six weeks after her initial presentation the patient's haemodynamic profile deteriorated and she underwent aortic valve replacement with a $23 \mathrm{~mm}$ Carbomedics prosthesis. Culture and chlamydia PCR of the excised valve was negative despite prolonged incubation. Subsequent PCR analysis of the excised native valve at the Central Public Health Laboratory (Colindale) was positive for Bartonella quintana. In addition retrospective serological testing of stored sera demonstrated rising antibody levels to B quintana.

At six month follow up the patient remained well and there were no clinical features of relapse. The C-reactive protein and ESR levels were normal and the doxycycline therapy was discontinued. In addition the patient's antinuclear and double stranded antibody titres had returned to normal.

\section{DISCUSSION}

Culture negative endocarditis is a significant clinical problem and it has been suggested that it accounts for between $5 \%$ and $30 \%$ of all cases of endocarditis. ${ }^{1}$ Previous antimicrobial treatment is by far the commonest cause of subsequently negative blood cultures. It has been shown that even a short course of oral antibiotic treatment can cause a long lasting suppression of bacterial activity; this effect may be partially overcome by performing additional serial blood cultures. ${ }^{2}$ When individuals with a history of antibiotic treatment are excluded from the total, the rate of "true" culture negative endocarditis is probably in the region of $5 \%{ }^{3}$ This figure includes slow growing fastidious bacteria and non-bacterial organisms, which require prolonged culture under stringent conditions or specialised serological testing for diagnosis (see box 1$) .4$

Bacteria of the genus bartonella are fastidious Gram negative rods and are being increasingly recognised as

Abbreviations: ESR, erythrocyte sedimentation rate; $P C R$, polymerase chain reaction 

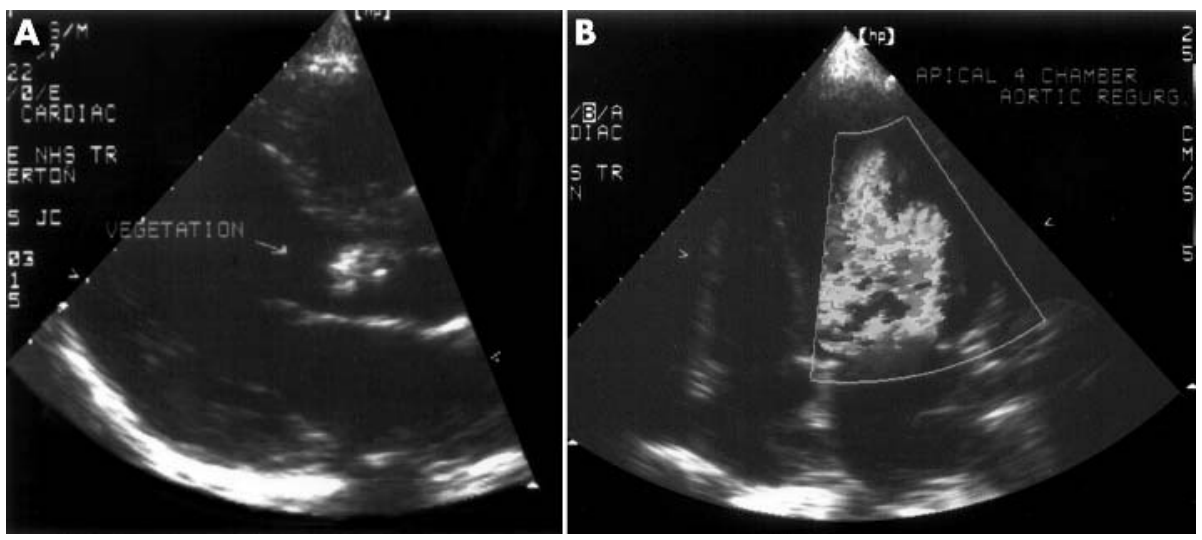

Figure 1 Transthoracic

echocardiogram demonstrating a large vegetation attached to the aortic valve (A: parasternal long axis view) and moderate to severe aortic regurgitation with colour flow mapping (B: apical four chamber view; shown in black and white).

human pathogens; currently 10 members have been identified, ${ }^{5}$ three of whom have been implicated as causes of endocarditis. B quintana is the agent responsible for trench fever, which afflicted approximately one million soldiers in World War I. ${ }^{6}$ The disease is associated with poor nutritional and sanitary conditions and the human body louse has been suggested as the disease vector. It has recently been identified as a cause of endocarditis in immunocompetent homeless men. ${ }^{7}$ Pre-existing native valve disease is not usually present and a history of alcohol abuse as well as male gender are often associated. ${ }^{8} B$ henselae has been reported as a cause of endocarditis and is usually associated with a history of contact with cats. ${ }^{9}$ B elizabethae has been recently implicated as the causative agent in a single case of endocarditis in an immunocompetent patient. ${ }^{10}$

The patient in this report has none of the previously described risk factors associated with bartonella infection, in that she was female, had no history of alcohol abuse or exposure to cats, and was not homeless. Interestingly she demonstrated serological positivity to Chlamydia spp, presumably due to cross reactivity, which has been previously reported $^{7}$ and may have resulted in misdiagnosis in the past. Indeed in our patient's case there was a history of exposure to bird droppings at the staff entrance to the supermarket and chlamydial infection was entertained in the differential diagnosis. In addition, as far as we are aware this is the first reported association of false positive double stranded

\section{Box 1: Factors responsible for "culture} negative" endocarditis

- Previous antibiotic treatment.

- Fungi and yeast.

- Fastidious organisms: Bartonella spp, legionella, mycobacterium, brucella, HACEK group (Haemophilus spp, Actinobacillus actinomycetemomitans, Cardiobacterium hominis, Eikenella corrodens, and Kingella kingae).

- Cell dependent organisms: Coxiella burnetti, Chlamydia spp, Mycoplasma spp.
DNA titres and bartonella infection. Blood culture isolation of the bartonella genus is difficult and the best methods for diagnosis are specific antibody serology and PCR analysis of excised valve tissue. PCR testing is a widely used molecular approach to disease diagnosis. However, the initial enthusiasm for PCR testing was hampered by the frequent tendency to false positive results due to sample contamination. This problem has been greatly reduced by the introduction of newer techniques and sample handling protocols. In addition PCR testing also amplifies DNA from dead organisms, rendering a result diagnostically correct but possibly a clinical false positive. Due to limited clinical experience there are as yet no firm antibiotic recommendations for treatment of Bartonella spp; fortunately the organism shows sensitivity to a wide range of antibiotics in vitro.

\section{Authors' affiliations \\ P Mann, F Nye, G Williams, A Walker, A Amadi, Aintree Cardiac Centre, University Hospital Aintree, Liverpool}

Correspondence to: Dr Paul Mann, Cardiothoracic Centre, Thomas Drive, Liverpool, L1 4 3PE, UK; Paull Mann@aol.com

Submitted 9 February 2003

Accepted 21 April 2003

\section{REFERENCES}

1 Watanakunakorn C, Burkert T. Infective endocarditis at a large community teaching hospital 1980-1990. A review of 120 episodes. Medicine (Baltimore) 1993;72:90-102.

2 Pazin GJ, Saul S, Thompson ME. Blood culture positivity, suppression by antibiotic therapy in patients with bacterial endocarditis. Arch Intern Med 1982;142:263-8.

3 Tunkel Ar, Kaye D. Endocarditis with negative blood cultures. N Engl J Med 1992;326:1215-17.

4 Van Scoy RE. Culture negative endocarditis. Mayo Clin Proc 1982;57:149-54

5 Maurin M, Raoult D. Bartonella (rochalimaea) quintana infections. Clin Microbiol Rev 1996;9:273-92.

6 Byam W. Trench fever. Lice and their menace to man. London: Oxford University Press, 1919:120-30.

7 Drancourt M, Mainardi JL, Brouqui $P$, et al. Bartonella quintana endocarditis in three homeless men. N Engl J Med 1995;332:419-23.

8 Raoult $D$, Fournier PE, Draincourt $M$, et al. Diagnosis of 22 new cases of bartonella endocarditis. Ann Intern Med 1996;125:646-52.

9 Holmes AH, Greenough TC, Balady GJ, et al. Bartonella henselae endocarditis in an immunocompetent adults. Clin Infect Dis 1995;21:1004-7.

10 Daly JS, Worthington MG, Brenner DJ, et al. Rochalimaea elizabethae isolated from a patient with endocarditis. J Clin Microbiol 1993;31:872-81. 